\title{
Microbiome in Oral Squamous Cell Carcinoma: Mechanisms and Signaling Pathways
}

\author{
Nurani Hayati ${ }^{1}$, Caesary Cloudya Panjaitan², Ferry Sandra ${ }^{3}$ \\ ${ }^{1}$ Department of Conservative Dentistry and Endodontic, Faculty of Dentistry, Universitas Prof. Dr. Moestopo, Jakarta, Indonesia \\ ${ }^{2}$ Department of Public Health, Faculty of Dentistry, Universitas Trisakti, Jakarta, Indonesia \\ ${ }^{3}$ Department of Biochemistry and Molecular Biology, Division of Oral Biology, Faculty of Dentistry, Universitas Trisakti, Jakarta, \\ Indonesia
}

Oral squamous cell carcinoma is part of head and neck squamous cell carcinoma which is the ultimate cause of morbidity and mortality in cancer. The alteration of microbial community in the saliva might act as a helpful marker for the prediction, detection and prognosis oral cancer, particularly the transition of cancer precursor lesion. There are three mechanisms of action of oral microbiota in cancer pathogenesis, chronic inflammation of bacterial stimulation, carcinogenesis by cytoskeletal rearrangements, and carcinogenic substances that produced by microorganisms. Changes in the composition of microbiota could therefore have the potential to be used as a significant oral biomarker to predict the pathological transition from oral epithelial precursor lesion to cancer.

Keywords: microbiome, oral cancer cellular proliferation, microorganism, oral cancer, oral squamous cell carcinoma

\section{Introduction}

Oral squamous cell carcinoma (OSCC), a part of head and neck squamous cell carcinoma (HNSCC), has a high recurrence rate with lymph node. ${ }^{1}$ Most of the OSCC cases were diagnosed at late stage, hence the risk of secondary primary tumors was higher. ${ }^{2-5}$ The 2019 death rate of oral and pharyngeal cancers was 10,860 cases in the United States of America, and tongue cancer was the main cause of death in oral cancer. ${ }^{6}$ Recently, the rates of oral cancers have increased in developed countries such as Japan with an estimated of 7,000 new cases and 3,000 deaths with the 2:1 male-to-female ratio. ${ }^{7}$ The 5 -year cancer survival rate was

Date of submission: February 4, 2020 Last Revised: June 1, 2020

Accepted for publication: June 2, 2020

Corresponding Author:

Ferry Sandra

Department of Biochemistry and Molecular Biology, Division of Oral Biology

Faculty of Dentistry, Universitas Trisakti, Jl. Kyai Tapa No.260

Jakarta, Indonesia

E-mail: ferry@trisakti.ac.id
$60-80 \%$, but with early detection, the survival rate will rise to $90 \% .{ }^{6,7}$ Meanwhile in Indonesia in 2014, 319 oral cancer cases were diagnosed over the past 15 years at Dharmais National Cancer Hospital. ${ }^{8}$ The Dharmais data showed that OSCC was most frequently found on the tongue, nearly $70 \%$ were advanced stage with bad prognosis and poor survival rates. ${ }^{8}$

Tobacco, betel quid, and alcohol are the primary risk factors for OSCC. ${ }^{1}$ About $74 \%$ of OSCC cases are caused by tobacco chewing with or without betel nut. Risk factors such as tobacco smoking, alcohol consumption, and poor oral hygiene can inevitably make the case worse. ${ }^{2}$ Not only chemical irritants such as cigarette and alcohol, but 
the dental prosthesis inflammation, human papillomavirus (HPV), and chronic periodontitis infection can also increase the risk of oral cancer. ${ }^{1}$

Chronic periodontal disease involves a pathological organism that will increase the risk of premalignant lesions and cancer. ${ }^{6}$ According to some studies, oral pre-cancers and cancers affect the composition of the oral microbiome. In vice versa, chronic inflammation of bacterial infection induces cell proliferation, mutagenesis, oncogene activation and angiogenesis as well. ${ }^{3}$ Helicobacter pylori is the first bacterium known as carcinogenic inducer. ${ }^{4}$ Additionally, Porphyromonas gingivalis is another pathogenic bacterium that frequently found in OSCC. OSCC patients mostly suffer from chronic periodontitis, resulting in chronic inflammatory OSCC cells. ${ }^{5}$ The relation between oral cancer-inducing factors and bacterial ecology is significant since alterations in oral microbiome have also been found in subjects consuming alcohol, betel quid and smoking. ${ }^{9}$ Besides $P$. gingivalis, OSCC is strongly associated with bacteria such as Streptococcus sp., Peptostreptococcus sp., Prevotella sp., and Capnocytophaga gingivalis, while Fusobacterium, Veillonella, Actinomyces, Clostridium, Haemophilus and Enterobacteriaceae are associated with precursor lesions of the epithelium. ${ }^{3}$

\section{Oral Microbiome in Healthy Individuals}

Actinomyces, Capnocytophaga, Eikenella, Eubacteria, Fusobacterium, Haemophilus, Lactobacterium, Leptotrichia, Neisseria, Porphyromonas, Prevotella, Propionibacterium, Peptostreptococcus, Streptococcus, Staphylococcus, Veillonella and Treponema exist in healthy people's oral cavity. Other than that, Firmicutes, Proteobacteria, Actinobacteria, Bacteroidetes and Fusobacteria are also prevalent oral microbial species in healthy individuals. ${ }^{10}$ Those commensal species live with the host immune defense systems in equilibrium. ${ }^{9}$ The hostmicrobiota co-dependence is preserved with host-provided nutrient-rich environment, while the commensal microbes secrete nutrient processing metabolites, avoid pathogenic microbe infections, and maintain homeostasis by stimulating immune responses. ${ }^{10}$ Streptococcus is the predominant oral microbiome healthy genus. ${ }^{4}$ Commensal and pathogenic bacterial organisms, by forming biofilm, circumvent the host immune response. Any disturbances of this equilibrium and reorganization of the microbial composition have been involved in various diseases such as, dental caries or periodontal diseases. ${ }^{9}$ Most of these microorganisms live in a symbiotic capacity in our oral cavity, establishing mutually beneficial relationships with the host. Not only they are not harmful, but these commensal populations can also keep pathogenic organisms under control by not allowing them to adhere to mucosal surfaces. ${ }^{11}$

Commensal bacteria with proper inhibitors may improve the effectiveness of immunotherapy. ${ }^{5}$ Compared with programmed death ligand 1 (PD-L1) specific antibody therapy, Bifidobacterium by oral administration controlled tumor growth with the same efficacy. Both the oral administration of Bifidobacterium and the PD-L1-specific antibody avoided tumor outgrowth. Bifidobacterium oral administration improves antitumor resistance and changes the effectiveness of control-point inhibitors as an immunotherapeutic agent by modifying the composition of intestinal microorganisms. ${ }^{12}$

\section{Oral Microbiome in Cancer Patients}

Oral microbial communities tend to vary from stable sites to malignant sites. For example, Streptococcus anginosus and Treponema denticola associate with multiple carcinomas of the upper gastrointestinal tract. ${ }^{13}$ In general, anginosal infection may be involved in the carcinogenesis of HNSCC. The DNA of S. anginosus was found in specimens of carcinoma tissue but not in samples of lymphoma, rhabdomyosarcoma, or leukoplakia. Dental plaque may act as a dominant reservoir of this bacterium. ${ }^{14,15}$

In the pre-cancerous oral lesions, leukoplakia, Bacteroidetes and Fusobacteria are substantially enriched, compared to normal contralateral tissue in the same patients. ${ }^{4,16}$ Meanwhile among patients with oral cancer, a substantial increase in the number of $C$. gingivalis, P. melaninogenica, and S. mitis among patients was reported. ${ }^{17}$

The incidence of cancer worldwide also refers to virus-related infections such as hepatitis viruses, HPV, and Epstein-Barr virus (EBV). ${ }^{4}$ Nonetheless, in order to put viral infections in perspective, it has been estimated that at least six human viruses, EBV, hepatitis B virus (HBV), hepatitis $\mathrm{C}$ virus (HCV), HPV, human T-cell lymphotropic virus (HTLV-1) and Kaposi's associated sarcoma virus (KSHV) lead to $10-15 \%$ of all cancers worldwide. ${ }^{18,19} \mathrm{HPV}$ has been known recently as an etiological factor that cause oropharyngeal cancer, however, infection which caused by 
HPV is not a main contributor to oral cancer, since the virus is infrequently found in oral cancers (only $2-4 \%$ of cases). ${ }^{20}$

\section{Pathogenesis of Oral Microorganisms in Cancer Development}

There are three mechanisms of oral microbiota in carcinogenesis. The first is the chronic inflammation bacterial stimulation. Such mechanism may results inflammatory mediators that may promote cells proliferation, angiogenesis activation and mutagenesis. With regard to the second mechanism, bacteria can influence carcinogenesis by cytoskeletal rearrangements, activation of nuclear factorkappaB (NF-kB), and cellular apoptosis inhibition. The third mechanism is carcinogenic substances that produced by microorganisms. ${ }^{21}$

\section{Stimulation of Chronic Inflammation}

Porphyromonas, Prevotella and Fusobacterium as anaerobic species in oral cavity have significant role for periodontal diseases and chronic inflammatory mechanism. These bacteria induce inflammatory mediators production which affect epithelial and endothelial cells, fibroblasts, and extracellular matrix. These periodontal pathogens may affect the body to produce various cytokines such as interleukin (IL)-1 $\beta$, IL-6, IL-17, IL-23, tumor necrosis factor (TNF)- $\alpha$, and matrix metalloproteinases (MMP)-8 and MMP-9. ${ }^{22}$

In response to lipopolysaccharide (LPS), the key element of Gram-negative bacteria walls, the neutrophils, fibroblasts, and mast cells in periodontium tissues may synthesize IL-1 $\beta$ (Figure 1). The IL-1 $\beta$ induces the development of osteoclasts and bone resorption ${ }^{23}$, resulting in alterations of sectional inflammatory in the periodontium. IL-1 $\beta$ also provokes the secretion of acute phase proteins, prostaglandins (PG), phospholipase A2, and proinflammatory cytokines such as TNF- $\alpha$, IL- 6 , and MMPs. ${ }^{5,24}$ Furthermore, IL- $1 \beta$ also encourages endothelial cells to produce Vascular Endothelial Growth Factor (VEGF) and many proangiogenic factors. ${ }^{25}$ IL-1 $\beta$ was also associated with lower E-cadherin expression, which promotes cell migration. ${ }^{26}$ Therefore, high level of IL- $1 \beta$ is associated to proliferation, more invasive and aggressive phenotype of a tumor. ${ }^{25,27}$

IL-6, which produced by the influence of LPS, IL-1 $\beta$ and TNF- $\alpha$, also causes bone resorption and enhances the synthesis of PGE2, acute phase proteins, and chemokines. ${ }^{1,28-32}$ IL-6 generates oxidative stress and may lead to a short-term aggregation of $\mathrm{H}_{2} \mathrm{O}_{2}$ in mitochondria, resulting in damage of mitochondrial..$^{33}$ IL-6 also affects the invasion and metastasis process by promoting MMPs expressions. Furthermore, IL- 6 increases the release of Intercellular Adhesion Molecules (ICAMs) and Endothelial Leukocyte Adhesion Molecules (ELAMs), that promote adhesion of endothelial cells to tumor cells, thereby influencing the spread of tumors. Most genes activated by IL-6 are involved in the progression of the cell cycle and apoptosis suppression. IL-6 may influence the development of cancer by affecting anti-apoptotic pathways as well. $5,31,34$

TNF- $\alpha$ is secreted due to various elements, including bacterial LPS. Furthermore, TNF- $\alpha$ highly induces the progression of reactive oxygen species (ROS), PG, leukotrienes, and MMPs. TNF- $\alpha$ also decreases the production of osteogenic cells and fibroblasts. Unlike high doses of TNF- $\alpha$ that associated with tumor destruction, tumor development is related to low doses of TNF- $\alpha{ }^{35,36}$ TNF- $\alpha$ induces tumor growth by activation of carcinogenic signaling pathways in epithelial cells, including Wnt and NF- $\kappa B .{ }^{28}$ TNF- $\alpha$ also has the ability of generating ROS to cause DNA damage. It has been shown that TNF- $\alpha$ affects motility and invasion processes by inducing MMPs to express and induce the development of various angiogenic factors such as IL-8 and VEGF. ${ }^{37}$

\section{Anti-apoptotic Mechanisms}

Epithelial cell responses to infection of $P$. gingivalis in both apoptosis and cell division. P. gingivalis can highly induce anti-apoptotic activity in primary cultures of gingival epithelial cells. ${ }^{14}$ Role of $P$. gingivalis infection in tumor development remains to be determined, however, there is a solid proof of its inherent ability to influence many aspects of epithelial cell signaling. $P$. gingivalis promotes intracellular signaling of Jak1/Akt/Stat3, which lead to inhibition of mitochondrial apoptosis pathway $^{6}$, which has been targeted for inducing apoptosis in oral cancer cell. ${ }^{38,39}$ It demonstrated the ability to lower the activity of p53 tumor suppressor ${ }^{40} P$. gingivalis induces significant phosphorylation and inhibition of Bad in the membrane of mitochondrial as well as enhancing Bcl2/Bax ratios. In addition, $P$. gingivalis also suppresses caspases, important apoptotic machinery proteins which has been targeted for inducing apoptosis in oral cancer cell as well. ${ }^{39}$

P. gingivalis may also triggered adhesion of purinergic receptor P2X7-ATP, which plays a significant role in 


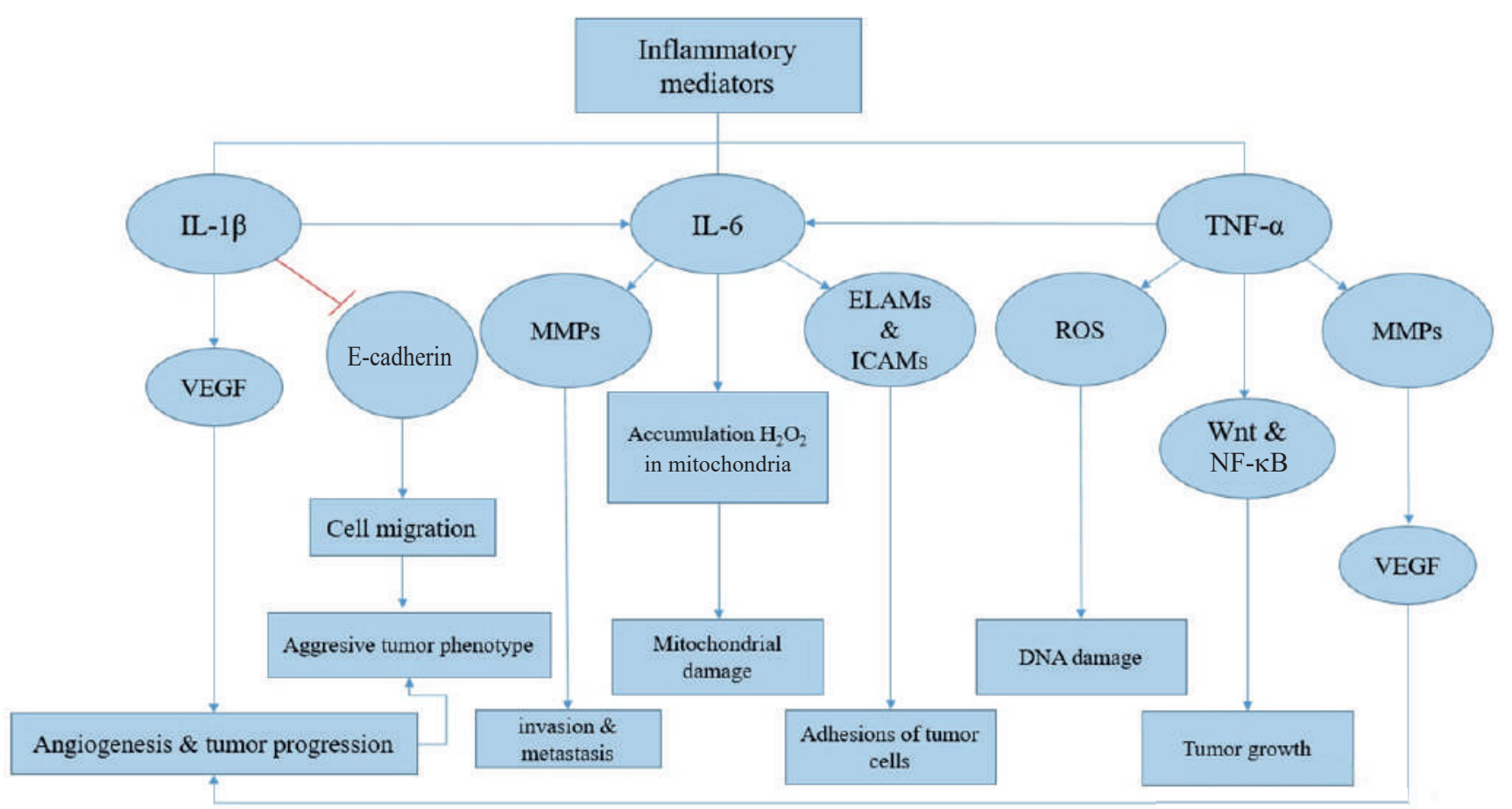

Figure 1. The mechanisms of chronic inflammation in carcinogenesis. Various inflammatory mediators induced by LPS bacteria may promote angiogenesis and tumor progression, aggressive tumor phenotype, invasion and metastasis, mitochondrial damage and adhesions of tumor cells.

neovascularization, metastasis, cell growth and secretion of inflammatory cytokines. This microorganism has shown its capability to release an anti-apoptotic enzyme nucleoside diphosphate kinase (NDK) that cleaves ATP and inhibits the activation of proapoptotic $\mathrm{P} 2 \mathrm{X} 7$ receptor (Figure 2), thus facilitating the signaling of ATP/P2X7. ${ }^{5}$ This NDK secretion may also regulate the cytosolic ATP-induced and ROS of mitochondrial which is an important mediator for the activation of transcription elements related to inflammation and the development of cancer. ${ }^{5,41}$ In addition, $P$. gingivalis produces gingipains, which actually are cysteine proteinases, that can break the MMP-9 pro-enzyme into its mature active form. ${ }^{5,21,40}$ Gingipains activation of MMP-9 induces degradation of the structure of the basal membrane, which encourages migration and invasion of cancer cells. ${ }^{5,42}$

Another oral microorganism, Fusobacterium nucleatum, may be the causative agent of oral squamous cell, pancreatic, and colorectal cancers. F. nucleatum infection modulates many anti-apoptotic pathways ${ }^{43}$, including Toll-like receptor (TLR) activation and NF$\kappa \mathrm{B}$ signaling (Figure 3) ${ }^{41}$ The most important part in the direct role of $F$. nucleatum in the carcinogenesis is the adhesin/invasin FadA, which adheres to E-cadherin on cancer cells and encourages the signaling of $\beta$-catenin. This pathway leads to increase Wnt transcriptional activity, pro-inflammatory cytokine activation, oncogenes, and cancer cells proliferation stimulation. ${ }^{5,44} \mathrm{~F}$. nucleatum also stimulates p38, leads to secretion of MMP-9 and MMP-13. Similar to MMP-9, MMP-13 also plays a significant role in tumor metastasis and invasion. ${ }^{42}$

\section{Carcinogenic Effect Substances}

Microorganisms play an important role in the metabolization of alcohol to acetaldehyde. ${ }^{5}$ Many types of oral microorganisms including streptococci S. Gordonii, S. Mitis, S. oralis, S. Salivary, S. sanguinis, and Candida yeasts, have the alcohol dehydrogenase (ADH) enzyme, that metabolizes alcohol to acetaldehyde with subsequent potential for oral cancer growth. ${ }^{45}$

Lactic acid is produced by certain oral bacteria of the genus Lactococcus, Lactobacillus, Streptococcus, Bifidobacterium, Leuconostoc and Pediococcus. Some species can produce more acids, for example Peptostreptococcus stomatis which produces acetic, butyric, isobutyric, isovaleric and isocaproic acids. ${ }^{5}$ The majorities of bacteria isolated from the OSCC tissues were 


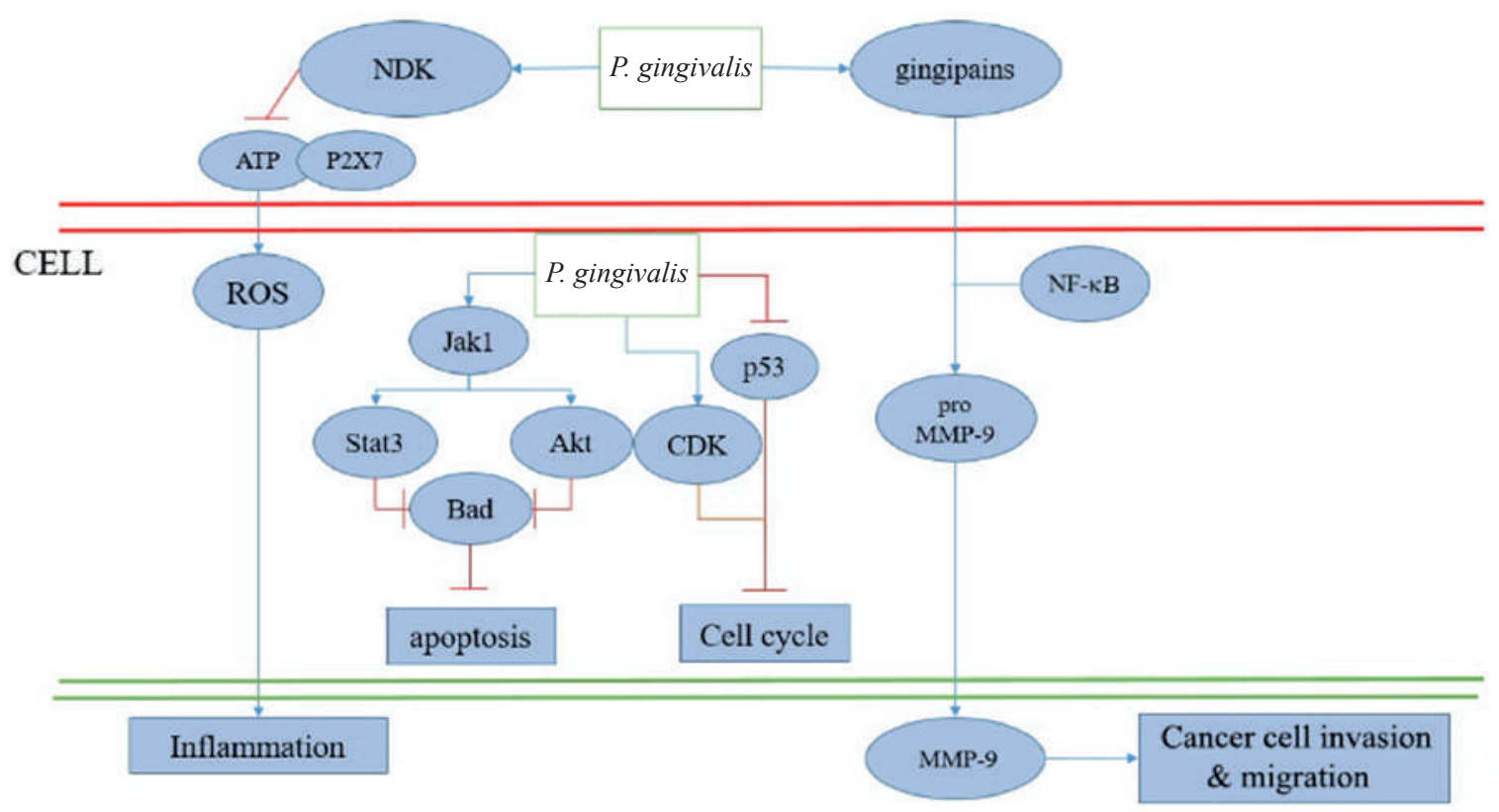

Figure 2. $P$. gingivalis can cause cell-cycle changes and apoptosis inhibition. Extracellular $P$. gingivalis may induce inflamation and promote cancell cell invasion and migration through gingipains proteinases. Intracellular $P$. gingivalis may inhibit apoptosis mechanism and disrupt cell cycle through activation of Jak1 and decrease the activity of p53.

saccharolytic and aciduric bacteria ${ }^{46}$ Such microorganisms may influence the $\mathrm{pH}$ reduction in the local environment by generating lactic acid. This acid production may enhance the acidic and hypoxic tumor microenvironment, thus increasing metastatic capability. ${ }^{47}$

Several oral microorganisms such as $P$. gingivalis, Prevotella intermedia, Aggregatibacter actinomycetemcomitans, and F. nucleatum contain volatile sulfur compounds (VSC), such as hydrogen sulfide $\left(\mathrm{H}_{2} \mathrm{~S}\right)$, dimethyl sulfide $\left(\left(\mathrm{CH}_{3}\right)_{2} \mathrm{~S}\right)$, dimethyl disulfide $\left(\mathrm{CH}_{3} \mathrm{SSCH}_{3}\right)$ and methyl mercaptan $\left(\mathrm{CH}_{3} \mathrm{SH}\right) .{ }^{5} \mathrm{H}_{2} \mathrm{~S}$ appears in saliva at the highest concentration, while $\mathrm{CH}_{3} \mathrm{SH}$ is the dominant compound in the gingival pockets. $\mathrm{H}_{2} \mathrm{~S}$ is known as genotoxic agent and may result in cumulative mutations or genomic instability. Increased expression of various $\mathrm{H}_{2} \mathrm{~S}$-producing enzymes in cancer cells has been observed, particularly in colon and ovarian cancers. ${ }^{48}$

It was also shown that $\mathrm{H}_{2} \mathrm{~S}$ promotes apoptosis in human gingival epithelial cells through mitochondrial pathway activation (Figure 4). ${ }^{49}$ Upon cell exposure to $\mathrm{H}_{2} \mathrm{~S}$, apoptosis was remarkably increased at 24 and 48 hours, ROS was enhanced, and depolarization of the mitochondrial membrane was disintegrated. Release of Cytochrome C, a small protein known to be related with the mitochondrial inner membrane involved in apoptosis initiation, increased significantly. Activities of Caspase- 9 and -3 were reported highly stimulated, while the activity of caspase- 8 remained low. In 48-hours the percentage of DNA-strand-break increased. ${ }^{48,49}$

Recent findings suggest that there are various mechanisms that lead to the effect of $\mathrm{H}_{2} \mathrm{~S}$ on cancer, consist of angiogenesis induction, control of mitochondrial bioenergetics, cell cycle progression acceleration, and anti-apoptosis functions. ${ }^{50}$ Studies showed that $\mathrm{H}_{2} \mathrm{~S}$ has proangiogenic effects by enhancing the expression of VEGF in both kidney and ischemic tissues. ${ }^{51}$ In addition, many evidence suggests that $\mathrm{H}_{2} \mathrm{~S}$ is an endogenous angiogenesis stimulator and promotes the growth and proliferation of tumor cells. Cystathionine $\beta$-synthase (CBS)-derived $\mathrm{H}_{2} \mathrm{~S}$ is used to induce angiogenesis and vasorelaxation, therefore promoting tumor growth in colon cancer. ${ }^{50,52}$

Growing evidence suggests that $\mathrm{H}_{2} \mathrm{~S}$ is involved in regulating mitochondrial function and cellular bioenergetics by direct donation of electrons and mitochondrial phosphodiesterases inhibition. ${ }^{50}$ CBS co-locates with mitochondrial markers in ovarian cancer cells, and silencing CBS reduces the intake of mitochondrial oxygen accompany with an increase the production of ROS..$^{53}$ In 


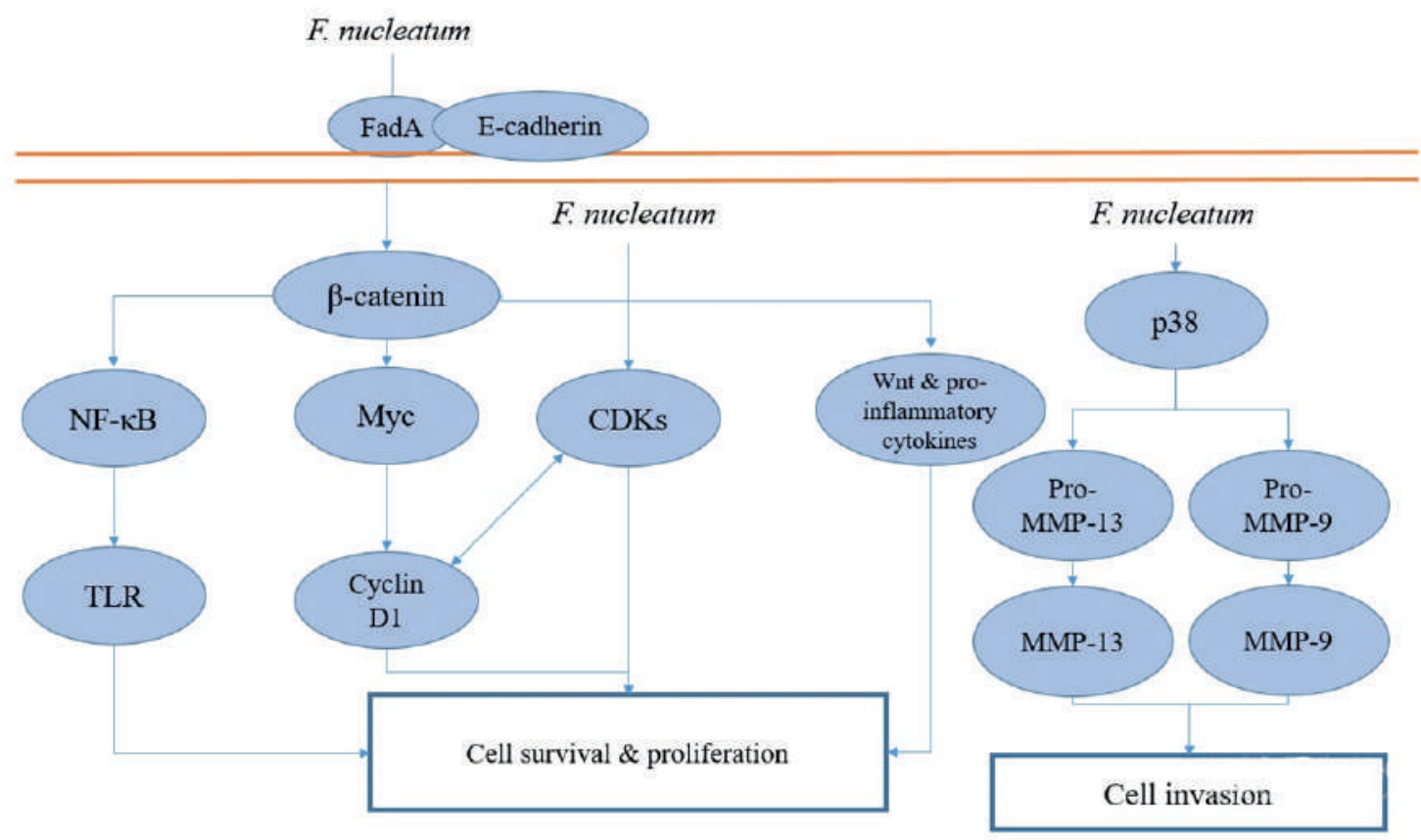

Figure 3. F. nucleatum can generate an oncogenic phenotype of epithelial cell. FadA will activating pathway of $\beta$-catenin, which leads to cell survival and proliferation. Intracellular F. nucleatum through p38 may cause MMP-9 and MMP-13 activation, which play an important part in cell invasion.

summary, CBS that generated from $\mathrm{H}_{2} \mathrm{~S}$ could sustain cellular bioenergetics in cancer cells, therefore promoting tumor growth and proliferation..$^{50}$ However, a recent study also shows that exogenous $\mathrm{H}_{2} \mathrm{~S}$ (NaHS, 200-500 $\mu \mathrm{M}$ ) might serve as a proliferative induced factor that accelerates the progression of the cell cycle in oral squamous cell carcinoma by increasing phosphorylation of protein kinase B (PKB)/ Akt and extracellular signal-regulated kinase (ERK). ${ }^{50,54}$

\section{Microbiology Alteration as Biomarker for Oral Diagnostic}

There are more than 500 species microorganisms in saliva of oral cancer patients, both cultivated and uncultivated varieties. ${ }^{3}$ Compared to the approximately 50 strains observed using standard experimental methods, this large data set reflects significant improvement. Changes in the microbial community (Peptostreptococcus, Bacillus, Parvimonas, Enterococcus, and Slackia) in the saliva could act as a convenient marker for predicting, and detecting oral cancer, particularly the epithelial precursor lesion-cancer transition. At least nine bacteria, including Alistipes, Bacteroids, Blautia, Clostridium, Dorea, Escherichia, Faecalibacterium, Megamonas, and Phascolarctobacterium, showed positive associations in the epithelial precursor lesion and cancer groups. In addition, the normal group detected a very different positive/negative correlation network, suggesting community alteration in the disease group. Such associations may indicate the microbial community changes in saliva, which could identify many combinations of microorganisms associated with oral cancer and epithelial precursor lesions. ${ }^{3}$ High salivary numbers of Capnocytophaga gingivalis, Prevotella melaninogenica and Streptococcus mitis can be used as OSCC diagnostic indicators. The involvement of on the salivary microbiota on OSCC has more severe effect than tobacco. ${ }^{17}$ The surfaces of OSCC display significantly elevated levels of Porphyromonas and Fusobacterium relative to healthy mucosa. ${ }^{55,56}$

\section{Conclusion}

There are three mechanisms of action of oral microbiota in cancer pathogenesis, chronic inflammation of bacterial stimulation, carcinogenesis by cytoskeletal rearrangements, and carcinogenic substances that produced by microorganisms. The alteration of microbial community in the saliva might represent as a convenient marker for 


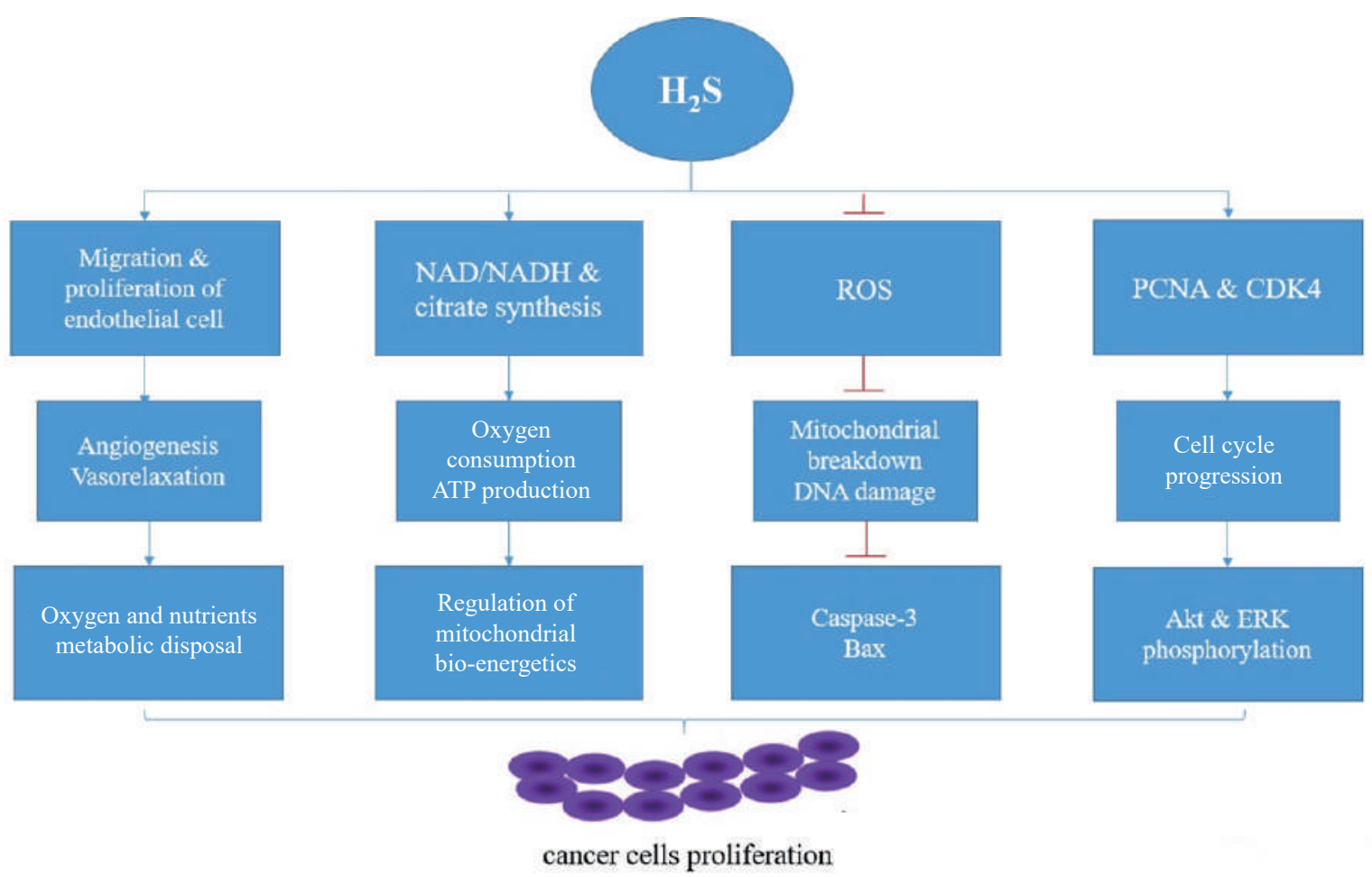

Figure 4. A schematic ilustration of $\mathrm{H}_{2} \mathrm{~S}$ in developing cancer cell proliferation. Induction of angiogenesis, regulation of mitochondrial bioenergetics, acceleration of cell cycle progression, and anti-apoptotic function contribute to the carconigenesis mechanisms of $\mathrm{H}_{2} \mathrm{~S}$. $\mathrm{H}_{2} \mathrm{~S}$ : hydrogen sulfide; NAD: oxidized form of nicotinamide adenine dinucleotide; NADH: reduced form of nicotinamide adenine dinucleotide; ATP: adenosine 5'-triphosphate; ROS: reactive oxygen species; Bax: B-cell lymphoma gene-2 associated X protein; PCNA: proliferating cell nuclear antigen; CDK4: cyclin-dependent kinase 4; ERK: extracellular signal-regulated kinase.

the prediction, detection, and prognosis of oral cancer, especially the epithelial precursor lesion-cancer transition.

\section{References}

1. Chattopadhyay I, Verma M, Panda M. Role of oral microbiome signatures in diagnosis and prognosis of oral cancer. Technol Cancer Res Treat. 2019; 18: 153303381986735 . doi: $10.1177 / 1533033819867354$.

2. Ferlay J, Shin HR, Bray F, Forman D, Mathers C, Parkin DM. Estimates of worldwide burden of cancer in 2008 : GLOBOCAN 2008. Int J Cancer. 2010; 127(12): 2893-2917.

3. Lee WH, Chen HM, Yang SF, Liang C, Peng CY, Lin FM, et al. Bacterial alterations in salivary microbiota and their association in oral cancer. Sci Rep. 2017; 7(1): 1-11. doi:10.1038/s41598-01716418-x.

4. Schmidt BL, Kuczynski J, Bhattacharya A, Huey B, Corby PM, Queiroz ELS, et al. Changes in abundance of oral microbiota associated with oral cancer. PLoS One. 2014;9(6): e98741. doi: 10.1371/journal.pone.0098741.

5. Karpinski TM. Role of oral microbiota in cancer development. Microorganisms. 2019; 7(1): 20. doi: 10.3390/ microorganisms 7010020 .
6. Takahashi Y, Park J, Hosomi K, Yamada T. Analysis of oral microbiota in Japanese oral cancer patients using $16 \mathrm{~S}$ rRNA sequencing. J Oral Biosci. 2019; 61(2): 120-8.

7. Warnakulasuriya S. Global epidemiology of oral and oropharyngeal cancer. J Oral Oncol. 2009; 45(4-5): 309-316.

8. Purwanto DJ, Soedarsono N, Reuwpassa JO, Adisasmita AC, Ramli M, Djuwita R. The prevalence of oral High-risk HPV infection in Indonesian oral squamous cell carcinoma patients. Oral Dis. 2020; 26(1): 72-80.

9. Lamont RJ, Jenkinson HF. Oral Microbiology at a Glance. Chichester, West Sussex: Wiley-Blackwell; 2010.

10. Zaura E, Keijser BJF, Huse SM, Crielaard W. Defining the healthy "core microbiome" of oral microbial communities. BMC Microbiol. 2009;12(9): 259. doi:10.1186/1471-2180-9-259.

11. Avila M, Ojcius DM, Yilmaz Ö. The oral microbiota: living with a permanent guest. DNA Cell Biol. 2009; 28(8): 405-11.

12. Sivan A, Corrales L, Hubert N, Williams JB, Aquino-Michaels K, Earley ZM, et al. Commensal bifidobacterium promotes antitumor immunity and facilitates anti - PD-L1 efficacy. Science. 2015; 350(6264): 1084-9.

13. Narikiyo M, Tanabe C, Yamada Y, Igaki H, Tachimori Y, Kato H, et al. Frequent and preferential infection of Treponema denticola, Streptococcus mitis, and Streptococcus anginosus in esophageal cancers. Cancer Sci. 2004; 95(7): 569-74. 
14. Meurman JH. Oral microbiota and cancer. J Oral Microbiol. 2010; 2: 5195. doi:10.3402/jom.v2i0.5195.

15. Shiga K, Tateda M, Salto S, Hori T, Sato I. Presence of Streptococcus infection in extra-oropharyngeal head and neck squamous cell carcinoma and its implication in carcinogenesis. Oncol Rep. 2001; 8(2): 245-8.

16. Amer A, Galvin S, Healy CM, Moran GP. The microbiome of potentially malignant oral leukoplakia exhibits enrichment for Fusobacterium, Leptotrichia, Campylobacter, and Rothia Species. Front Microbiol. 2017; 8: 2391. doi: 10.3389/fmicb.2017.02391.

17. Mager D, Haffajee A, Devlin P, Norris C, Goodson J. The salivary microbiota as a diagnostic indicator of oral cancer: A descriptive, non-randomized study of cancer-free and oral. J Transl Med. 2005; 3: 27. doi: 10.1186/1479-5876-3-27.

18. Syrjänen S, Syrjänen K. HPV in head and neck carcinomas: different HPV profiles in oropharyngeal carcinomas - why? Acta Cytol. 2019; 63(2): 124-42. doi: 10.1159/000495727.

19. Yete S, Souza WD, Saranath D. High-risk human papillomavirus in oral cancer : clinical implications. Oncology. 2017; 94(3): 133-41.

20. Gillison ML. Human papillomavirus - associated head and neck cancer is a distinct epidemiologic, clinical, and molecular entity. Semin Oncol Elsevier. 2004; 31(6): 744-54.

21. Zhang Y, Wang X, Yan F. Human oral microbiota and its modulation for oral health. Biomed Pharmacother. 2018; 99: 883-93.

22. Szkaradkiewicz AK, Karpiński TM. Microbiology of chronic periodontitis. J Biol Earth Sci. 2013; 3(1): 14-20.

23. Afifah E, Mozef T, Sandra F, Arumwardana S, Rihibiha DD, Nufus $\mathrm{H}$, et al. Induction of matrix metalloproteinases in chondrocytes by interleukin IL-1 $\beta$ as an osteoarthritis model. J Math Fund Sci. 2019; 51(2): 103-11.

24. Hou L, Liu C, Liu B, Lin SJ, Liao CS, Rossomando EF. Interleukin1B, clinical parameters and matched changes of biopsied gingival tissue from periodontitis patients. J Periodont Res. 2003; 38(3): 247-54.

25. Carmi Y, Dotan S, Rider P, Kaplanov I, White MR, Baron R, et al. The role of IL-1 $\beta$ in the early tumor cell-induced angiogenic response. J Immunol. 2013; (190): 3500-9.

26. Wang F, Liu H, Liu S, Tang S, Yang L, Feng G. SHP-2 promoting migration and metastasis of MCF-7 with loss of E-cadherin, dephosphorylation of FAK and secretion of MMP-9 induced by IL-1 b in vivo and in vitro. Breats Cancer Res Treat. 2005; 89(1): 5-14.

27. Voronov E, Shouval DS, Krelin Y, Cagnano E, Benharroch D, Iwakura $\mathrm{Y}$, et al. IL-1 is required for tumor invasiveness and angiogenesis. Prod Natl Acad Sci. 2002; 100(5): 2645-50.

28. Sandra F, Kukita T, Tang QY, lijima T. Cafeic acid inhibits NFkB activation of osteoclastogenesis signaling pathway. Indones Biomed J 2011; 3(3): 216-22.

29. Sandra F, Kukita T, Muta T, Iijima T. Caffeic acid inhibited receptor activator of nuclear factor $\mathrm{\kappa B}$ ligand (RANKL)-tumor necrosis factor (TNF) $\alpha$-TNF receptor associated factor (TRAF) 6 induced osteoclastogenesis pathway. Indones Biomed J. 2013; 5(3): 173-8.

30. Ketherin, Sandra F. Osteoclastogenesis in periodontitis: signaling pathway, synthetic and natural inhibitors. Mol Cell Biomed Sci. 2018; 2(1): 11-8.

31. Gabay C. Interleukin-6 and chronic inflammation. Arthritis Res Ther. 2006; 8(Suppl 2): S3. doi: 10.1186/ar1917.
32. Sandra F, Briskila J, Ketherin. RANKL and TNF-a-induced JNK/ SAPK osteoclastogenic signaling pathway was inhibited by caffeic acid in RAW-D cells. Indones J Cancer Chemoprevent. 2018; 9(2): 63-7.

33. Murata M, Thanan R, Ma N, Kawanishi S. Role of nitrative and oxidative DNA damage in inflammation-related carcinogenesis. J Biomed Biotechnol. 2012;2012: 623019. doi: 10.1155/2012/623019.

34. Haura EB, Turkson J, Jove R. Mechanisms of disease: insights into the emerging role of signal transducers and activators of transcription in cancer pathways. Nat Rev Clin Oncol. 2005; 2(6): 315-24.

35. Rivas MA, Carnevale RP, Proietti CJ, Rosemblit C, Beguelin W, Salatino M, et al. TNF $\alpha$ acting on TNFR1 promotes breast cancer growth via p42/P44 MAPK, JNK, Akt and NF-kB-dependent pathways. Exp Cell Res. 2008; 314(3): 509-29.

36. Sandra F, Matsuki NA, Takeuchi H, Ikebe T, Kanematsu T, Ohishi M, Hirata M. TNF inhibited the apoptosis by activation of Akt serine/threonine kinase in the human head and neck squamous cell carcinoma. Cell Signal. 2002; 14(9): 771-8.

37. Yan B, Wang H, Rabbani ZN, Zhao Y, Li W, Yuan Y, et al. Tumor necrosis factor-A is a potent endogenous mutagen that promotes cellular transformation. Cancer Res. 2006; 66(24): 11565-71.

38. Wicaksono BD, Tangkearung E, Sandra F. Brucea javanica Leaf Extract Induced Apoptosis in Human Oral Squamous Cell Carcinoma (HSC2) Cells by Attenuation of Mitochondrial Membrane Permeability. Indones Biomed J. 2015; 7(2): 107-10

39. Rizal MI, Sandra F. Brucea javanica Leaf Extract Activates Caspase-9 and Caspase- 3 of Mitochondrial Apoptotic Pathway in Human Oral Squamous Cell Carcinoma. Indones Biomed J. 2016; 8(1): 43-8

40. Inaba $\mathrm{H}$, Sugita $\mathrm{H}$, Kuboniwa $\mathrm{M}$, Iwai $\mathrm{S}$, Hamada $\mathrm{M}$, Noda $\mathrm{T}$, et al. Porphyromonas gingivalis promotes invasion of oral squamous cell carcinoma through induction of proMMP9 and its activation. Cell Microbiol. 2014; 16(1): 131-45.

41. Chen R, Alvero AB, Silasi D, Mor G. Inflammation, cancer and chemoresistance: taking advantage of the toll-like receptor signaling pathway. Am J Reprod Immunol. 2006; 57(2): 93-107.

42. Gholizadeh P, Eslami H, Yousefi M, Asgharzadeh M, Aghazadeh M, Kafil HS. Role of oral microbiome on oral cancers, a review. Biomed Pharmacother. 2016; 84: 552-8.

43. Gholizadeh P, Eslami H, Kafil H. Carcinogenesis mechanisms of Fusobacterium nucleatum. Biomed Pharmacother. 2017; 89: 91825.

44. Rubinstein MR, Wang X, Liu W, Hao Y, Cai G, Han YW. Fusobacterium nucleatum promotes colorectal carcinogenesis by modulating E-cadherin/B-catenin signaling via its FadA Adhesin. Cell Host Microbe. 2013; 14(2): 195-206.

45. Pavlova SI, Jin L, Gasparovich SR, Tao L, Tao L. Multiple alcohol dehydrogenases but no functional acetaldehyde dehydrogenase causing excessive acetaldehyde production from ethanol by oral streptococci Printed in Great Britain. Microbiology. 2013; 159(7): 1437-46.

46. Hooper SJ, Crean S, Fardy MJ, Lewis MAO, Spratt DA, Wade WG, et al. A molecular analysis of the bacteria present within oral squamous cell carcinoma. J Med Microbiol. 2007; 56(12): $1651-9$.

47. Lunt SJ, Chaudary N, Hill RP. The tumor microenvironment and metastatic disease. Clin Exp Metastasis. 2009; 26(1): 19-34.

48. Milella L. The negative effects of volatile sulphur compounds. J Vet Dent. 2015; 32(2): 99-102. 
49. Calenic B, Yaegaki K, Murata T, Imai T, Aoyama I, Sato T, et al. Oral malodorous compound triggers mitochondrial-dependent apoptosis and causes genomic DNA damage in human gingival epithelial cells. J Periodont. 2010; 45(1): 31-7.

50. Wu D, Si W, Wang M, Lv S, Ji A, Li Y. Hydrogen sulfide in cancer: Friend or foe? Nitric Oxide. 2015; 50: 38-45.

51. Wang M, Ding Y, Chen Y. The hydrogen sulfide donor NaHS promotes angiogenesis in a rat model of hind limb ischemia. Antioxid Redox Signal. 2010; 12(9): 1065-77.

52. Szabo C, Coletta C, Chao C, Módis K, Szczesny B, Papapetropoulos A. Tumor-derived hydrogen sulfide, produced by cystathionine$\beta$-synthase, stimulates bioenergetics, cell proliferation, and angiogenesis in colon cancer. Prod Natl Acad Sci. 2013; 110(30):
12474-9.

53. Bhattacharyya S, Saha S, Giri K, et al. Cystathionine beta-synthase (CBS) contributes to advanced ovarian cancer progression and drug resistance. PLoS One. 2013; 8(11); e79167. doi: 10.1371/journal. pone.0079167.

54. Ma Z, Bi Q, Wang Y. Hydrogen sulfide accelerates cell cycle progression in oral squamous cell carcinoma cell lines. Oral Dis. 2014; 21(2): 156-62.

55. Gaonkar P, Patankar S, Tripathi N, Sridharan G. Oral bacteria flora and oral cancer : the possible link? J oral Maxillofac Pathol. 2018; 22(2): 234-8.

56. Whitmore SE, Lamont RJ. Oral bacteria and cancer. PLoS Pathog. 2014; 10(3): 1-4. doi:10.1371/journal.ppat.1003933. 